\title{
Current perspectives of mitochondrial dysfunction and associated diseases
}

\begin{abstract}
Mitochondria are morphologically dynamic cell organelles that meet majority of energy requirements of cells. Genetic or acquired mitochondrial dysfunctions may result into significant abnormalities in regular cell functions. Being the most active cell type, hepatocytes possess a larger number of mitochondria for higher energy necessities and hence more vulnerable to mitochondrial dysfunction. Clinically mitochondrial damage has been found to cause various diseases, most of which are untreatable. Heteroplasmic nature of mitochondrial DNA makes diagnosis and treatment of mitochondrial disorders more challenging. Although exercise and gene therapies are extensively being studied, effective treatment strategies for mitochondrial dysfunction disorders are still lacking. This review summarizes clinically well characterized mitochondrial dysfunctional disorders and a few key approaches being used and/ or under investigation for diagnosis and treatment of the diseases. Comprehensive catalogs and indices to describe clinically significant mitochondrial aberrations are still under construction. Databases comprised of these catalogs and indices will help to develop targeted diagnostic and therapeutic approaches for mitochondrial disorders.
\end{abstract}

Volume 3 Issue | - 2016

\author{
Nidhi Gupta \\ Centrillion Technologies, USA
}

Correspondence: Nidhi Gupta, Centrillion Technologies, 2500 Faber Place, Palo Alto, CA, USA, Tel 7I84065732,

Email nidhi_in84@yahoo.com

Received: November 17, 2015 | Published: January 06, 2016

Keywords: diagnosis of mitochondrial disorders, dysfunctional disorders, hepatopathies, mitochondria, mitochondrial genome, mitochondrial disorder treatment, mitochondrial membrane

Abbreviations: CPEO, chronic progressive external ophthalmoplegia; KSS, kearns-sayre syndrome; MELAS, mitochondrial encephalopathy lactic acidosis stroke-like episodes; NARP, neuropathy ataxia retinitis pigmentosa; LHON, leber's hereditary optic neuropathy; MERRF, myoclonic epilepsy and ragged red fibers; iPSC, induced pluripotent stem cells; FIAU, fialuridine; d4T, stavudine; AZT, zidovudine; ddl, didanosine

\section{Introduction}

Mitochondria are intracellular organelles that serve as the metabolic powerhouses of cells since they are their primary source of ATP. ${ }^{1}$ Mitochondria play a crucial role in maintaining the high energy requirements of certain cells types such as the hepatocytes, which have more number of mitochondria per cell than most other cell types like small lymphocytes. ${ }^{2}$ Besides energy generation, several other characteristics of mitochondria make these essential for cells. Mitochondria are also involved in signalling, ${ }^{3,4}$ cellular differentiation ${ }^{5}$ and cell death. ${ }^{6}$

\section{Morphological dynamics of mitochondria}

Among intracellular organelles, mitochondria are structurally unique in that their surface consists of an outer and inner membrane. Both membranes create two compartments in the mitochondria called inter-membrane space and inner matrix. Integrity of inner and outer membranes of mitochondria is critical for mitochondrial function and is strictly regulated through interactions between different cellular proteins. ${ }^{7}$ Both mitochondrial membranes differ from each other in composition, properties and function. ${ }^{1}$ Though basic structure of phospholipids bilayer in the mitochondrial membranes is similar to the cell membranes, phospholipids compositions of the membranes of mitochondria differ from other membranes. ${ }^{8}$ Outer membrane of mitochondria contains integral membrane proteins called porins, which allow large sized molecules to freely diffuse across the outer membrane of mitochondria. ${ }^{9}$ Other proteins on the outer membrane, such as translocase, allow the movement of larger sized proteins to actively move across the membrane. ${ }^{10}$ Other essential mitochondrial outer membrane proteins like TOM20 and TOM 22 provide the negative charges to the outer membrane of mitochondria. ${ }^{11}$ The inner membrane of mitochondria is extensively folded into cristae, which results in increased total surface area of the inner membrane compared to outer mitochondrial membrane. ${ }^{12}$ Inner mitochondrial membrane closely resembles the bacterial membranes in their lipid composition, characterized by increased un saturation because of presence of low levels of triglycerides. ${ }^{13}$ Inner membrane is also freely permeable to some molecules, such as oxygen, carbon dioxide, and water, and is associated with numerous essential proteins and enzymes which are involved in aerobic respiration. ${ }^{14}$ Together these proteins constitute respiratory complexes that are organized in specific order in inner membrane of mitochondria and take part in synthesis of ATP by mitochondria. ${ }^{15}$ In spite of their size and complexity, mitochondria are extremely dynamic cell organelles and by processes of fusion, fission and movement across the cytoskeletal structure they change their shape and size frequently. ${ }^{16}$ Mitochondria can divide independent of cell division and also increase in number in cells in response to the energy needs of the cell. ${ }^{17,18}$ Frequent fission and fusion of mitochondria in cells permits exchange of mitochondrial genome between fusing mitochondria and hence provide an efficient means of homeostasis and inter-mitochondrial DNA complementation. This allows mitochondria to be morphologically highly polymorphic ${ }^{12}$ and functionally independent within cells. ${ }^{17}$ Different proteins play different roles in regulating morphological remodeling of mitochondria. ${ }^{19}$ Several proteins such as Fis $1 / \mathrm{Mdv}^{20}$ or Drp1/Dnm1 ${ }^{21,22}$ are localized on outer membrane of mitochondria and regulate fission of mitochondria. Once initiated, fission of mitochondria has been shown to take place in approximately 3 hours. ${ }^{23}$ Other proteins like Mgm1/OPA1 ${ }^{24,25}$ 
and Fzo1p/Mfn $1 \& 2^{26}$ are localized on inner or outer membrane of mitochondria and involved in fusion of mitochondria.

\section{Mitochondrial genome}

Human mitochondrial genome is approximately 16,600 base pairs long and codes for 37 genes $^{27,28}$ which includes expression of 13 genes for respiratory complexes subunits, 22 for mitochondrial t-RNA and 2 for r-RNA. ${ }^{29}$ Mitochondrial protein expression has dual genetic origin, that is, expression from cell nucleus and mitochondrial genome. Many of the vital structural and functional proteins of mitochondria are expressed from cell nucleus, and are transported to mitochondria via specific translocations and signal sequences. Each mitochondrion has 5-12 circular DNA copies, and unique metabolic systems enclosed within double membranes. ${ }^{30}$ Mitochondria also possess own DNA replication machinery, which allows the mitochondrial DNA replication in inner matrix of mitochondria independent of cell nuclear DNA division. In contrast to cell nuclear DNA polymerase, which has proof-reading exonuclease mechanism that assists in repairing the errors or mis-matches occurred while DNA replication, ${ }^{31}$ mitochondrial DNA polymerase lacks nucleotide excision repair mechanisms $^{32}$ which results into accumulations of mis-matches and errors in mitochondrial DNA that take place during the DNA replication process or by radiation or chemical damage..$^{33}$ Even though a few kinds of mitochondrial DNA repair pathways, such as base excision repair, ${ }^{34}$ have been shown effective in mitochondrial genome repair, lack of other efficient proof reading and editing pathways and close proximity to reactive oxygen species from respiration complexes on inner membrane of mitochondria leads to increased damage and accumulation of mutations in mitochondrial DNA relative to nuclear DNA, which further give rise to heteroplasmic mitochondrial DNA. ${ }^{35}$ Penetrance and severity of expression of some mitochondrial disorders can be determined by proportion of accumulated mitochondrial DNA mutations. Some severe mutations may also cause cell, organ or organism death. However, milder mutations and heterogeneity can permit survival. ${ }^{36}$ Moreover, introduction of normal mitochondrial DNA to mitochondria with mutated DNA by dynamic networking allows an efficient mechanism of mitochondria survival..$^{37}$ In addition, random distribution of mitochondria among daughter cells during cell division causes delivery of different proportions of mitochondria containing normal and mutant mitochondrial DNA to each daughter cell which further allows mitochondrial disease free survival of cells and organs. Mitochondrial DNA sequence heteroplasmy has also been used to determine lineages. ${ }^{38}$ Despite the rearrangement of genes in mitochondrial DNA, comparison of DNA sequences in different species has shown high levels of homology. Concurrently, mitochondrial DNA sequences from different species can also be distinguished from each other on basis of non-homologous and rearranged mitochondrial DNA regions. ${ }^{39}$

\section{Mitochondrial regulation of cell apoptosis}

Apoptosis is programmed cell death, which helps in cell number regulation or elimination of unwanted and potentially dangerous or diseased cells. ${ }^{40}$ Apoptosis is tightly regulated by several types of signaling proteins and cascades. Proteolytic activity of caspase protein family plays an important role in apoptosis. ${ }^{41}$ Several mitochondrial proteins have been identified with various biochemical and molecular studies, which have a complex role in direct activation of cellular apoptotic programs in mammalian cells. ${ }^{42}$ The outer mitochondrial membrane permeabilization also triggers the caspase proteases activation. Various pro-apoptotic proteins released from inter-membrane space of mitochondria, such as cytochrome c, also promotes caspase activation. ${ }^{43}$ Consequently, integrity of outer membrane of mitochondria is highly regulated through complex interactions between numerous pro- and anti-apoptotic proteins in cells. ${ }^{44}$ Injury to the outer membranes of mitochondria also results in to mitochondrial dysfunction and causes cell death regardless of the activity of signaling cascades, though sometimes under some pathophysiological conditions cells can survive this damage. ${ }^{45}$

\section{Mitochondrial dysfunctional diseases}

Recently mitochondrial dysfunctions have been found related to an array of human diseases such as hepatic mitochondrial disorders, ${ }^{46}$ cardiac dysfunction ${ }^{47}$ and autism. ${ }^{48}$ Both nuclear and mitochondrial genome mutations encoding mitochondrial proteins contribute to mitochondrial dysfunction. ${ }^{49}$ Many factors including a threshold effect, and segregation and clonal expansion govern the penetrance of mitochondrial disease. Although with ageing accumulation of mutations increases, heteroplasmic mitochondrial DNA allow appropriate mitochondrial functions in cells until the ratio of mutated mitochondrial DNA to normal mitochondrial DNA increase significantly. At this threshold level clinical symptoms of mitochondrial dysfunction begin to appear. ${ }^{50}$ Though there is random segregation of mitochondria into two daughter cells, clinical indicators of mitochondrial disorders may express when mutant load in a specific cell type exceeds the pathogenic threshold. In addition, clonal expansion of specific type of mutated mitochondrial DNA molecules may also cause amplified risk for the manifestation of mitochondrial disorders. ${ }^{51}$ Many types of mutations are feasible in mitochondrial DNA. Point mutations may occur in protein-coding genes during mitochondrial DNA replication, and can cause major mitochondrial functional defects. Though being heteroplasmic, these mutations tend to be recessive; however increased levels of these mutations beyond threshold levels, may have deleterious effect on functions of mitochondria. ${ }^{52}$ Large-scale deletions and rearrangements of DNA are another kind of mitochondrial DNA anomalies. ${ }^{53}$ Aged tissues and individuals with neurodegenerative diseases have shown an exponential accumulation of such deletions in mitochondrial DNA. ${ }^{53,54}$ Currently no therapeutic method is available to replace or correct these dysfunctional mitochondria in cells. A database compiling comprehensive description and clinical properties of all known pathogenic and non-pathogenic mitochondrial DNA mutations is still under construction. ${ }^{55}$ Population and mitochondrial genome based studies have revealed that mitochondrial diseases are relatively common. ${ }^{56}$ Founder effects and genetic bottlenecks in some geographic areas can cause under- or over-representation of specific mitochondrial DNA disorders. ${ }^{57}$ Studies based on childhood and adult mitochondrial syndromes suggest that prevalence of mitochondrial disorders is approximately 1 in 5000, and could be much higher. ${ }^{57}$ Increased accumulation of mitochondrial mutations with aging also causes increased genetic and clinical manifestations of mitochondrial disorders.58 However, the age of onset is also governed by the severity of the biochemical defect caused by mutations. ${ }^{59}$ Several other nuclear genetic or environmental factors also play an important role in expression of disease. ${ }^{60}$ Mitochondrial DNA mutations associated clinical symptoms are extremely variable and can express at any stage in life (Table 1). There is an increase in evidence supporting the association of mitochondrial DNA mutations to ageing, neurodegeneration, and tumorigenesis. ${ }^{58}$ Following are a few examples of some mitochondrial diseases that show early onset in infancy or childhood. 
Table I Summary of mitochondrial dysfunctional diseases

\begin{tabular}{|c|c|c|c|}
\hline Age of onset & Disease & Causes & Effect \\
\hline \multirow{3}{*}{$\begin{array}{l}\text { Early-onset } \\
\text { in infancy or } \\
\text { childhood }\end{array}$} & Leigh syndrome & Mitochondrial DNA defects & Progressive neurodegeneration \\
\hline & Depletion syndrome & $\begin{array}{l}\text { Decrease in copy number of } \\
\text { mitochondrial DNA. }\end{array}$ & Muscle weakness; progressive encephalopathy; liver failure \\
\hline & Kearns-Sayre syndrome & $\begin{array}{l}\text { Single or large-scale deletions } \\
\text { in mitochondrial DNA }\end{array}$ & $\begin{array}{l}\text { Multisystem failure; external ophthalmoplegia; neurological } \\
\text { complications; cardiomyopathy; dysphagia. }\end{array}$ \\
\hline \multirow{3}{*}{$\begin{array}{l}\text { Late-onset in } \\
\text { childhood or } \\
\text { adult life }\end{array}$} & $\begin{array}{l}\text { Chronic progressive external } \\
\text { ophthalmoplegia }\end{array}$ & $\begin{array}{l}\text { Large-scale mitochondrial } \\
\text { DNA deletions }\end{array}$ & Paralysis of the eye muscles \\
\hline & $\begin{array}{l}\text { Neuropathy, ataxia, and retinitis } \\
\text { pigmentosa }\end{array}$ & $\begin{array}{l}\text { Maternally inherited } \\
\text { mitochondrial DNA defects }\end{array}$ & Neuropathy; seizures; dementia \\
\hline & $\begin{array}{l}\text { Myoclonic epilepsy and ragged } \\
\text { red fibers }\end{array}$ & $\begin{array}{l}\text { Point mutations in } \\
\text { mitochondrial DNA }\end{array}$ & Neurodegenerative disorders \\
\hline
\end{tabular}

Leigh syndrome: Progressive neurodegenerative disorder due to severe failure of oxidative metabolism. Disease is caused by various mitochondrial DNA defects. ${ }^{61}$

Depletion syndrome: Depletion syndrome found in several organs depending on specific tissues with mitochondrial DNA depletion, resulting in muscle weakness, progressive encephalopathy or liver failure..$^{55}$ Organ transplantation can be therapeutically effective for the patients because of tissue specific nature of the disorder. Such syndromes would also respond well to potential the tissue specific mitochondria replacement therapy, if developed, where healthy and functional mitochondria could be delivered to cells with depleted mitochondrial DNA.

Kearns-Sayre syndrome (KSS): Multisystem disorder, caused by single or large-scale deletions [49], including progressive external ophthalmoplegia, neurological complications, cardiomyopathy deafness, short stature and dysphagia.

Following are a few examples of some mitochondrial disorders that show late onset in childhood or adult life. mutations in protein coding regions of mitochondria

a. Mitochondrial encephalopathy, lactic acidosis, and strokelike episodes (MELAS): brain and visual field defects resulting in severe stroke-like episodes with seizures, encephalopathy, vomiting, migraine like headaches, ataxia, and cognitive impairment. Several mitochondrial DNA mutations affecting protein-encoding genes or mitochondrial t-RNA expressing genes cause MELAS. ${ }^{61,62}$

b. Chronic progressive external ophthalmoplegia (CPEO): progressive paralysis of the eye muscles caused by single or multiple large-scale mitochondrial DNA deletions. ${ }^{49,54}$

c. Neuropathy, ataxia, and retinitis pigmentosa (NARP): neuropathy including developmental delay, seizures, and dementia. It has been found that patients with higher mutant load have an early onset in childhood because of maternally inherited mitochondrial DNA defects. ${ }^{36}$ This reflects the importance of the levels of heteroplasmy influencing the penetration of mitochondrial disorders. d. Leber's hereditary optic neuropathy (LHON): damage to retinal ganglion cells of the optic nerve leading to visual loss. ${ }^{63,64}$ It is caused by homoplasmic mitochondrial DNA mutations, found more in males compared to females.

e. Myoclonic epilepsy and ragged red fibers (MERRF): progressive, neurodegenerative disease caused by point mutations in mitochondrial DNA. ${ }^{65}$

Instead of location and size of deleted regions, the levels and distribution of deleted mitochondrial DNA in tissues and organs are more important factors in determination of clinical symptoms. ${ }^{66}$ In majority of cases, mutations in mitochondrial DNA impairs the mitochondrial function, which further deprives the cells of its main source of energy required for its optimal function. This results into cell function impairment or death causing loss of tissue or organ function depending on levels of damage occurred at cellular levels.

Besides inheritance of mitochondrial DNA defects, environmental factors may also damage the mitochondria. Several toxic substances internalized by cells can directly or indirectly damage the mitochondria.

\section{Mitochondrial hepatopathies}

Hepatocytes require, and contain the highest density of mitochondria of all human cell types because of the high metabolic activity. Therefore, hepatocytes are more vulnerable to disorders that affect mitochondrial functions. ${ }^{67}$ Disorders affecting normal mitochondrial activities directly regulate hepatocellular metabolism causing in impaired liver function, steatosis and cell death. Clinically, genetic and more frequently drug and alcohol-induced mitochondrial damages have been found to cause severe liver diseases, which ultimately result in to liver failure and death (Table 2) ${ }^{68,69}$ Because of lack of distinct clinical symptoms, the diagnosis of mitochondrial hepatopathies is usually poor and current medical treatments available are largely ineffective. Nevertheless, numerous specific molecular defects (including mutations, deletion or rearrangement of mitochondrial DNA) in hepatic mitochondria have been recognized in recent years. Mitochondrial hepatopathies can be acquired due to alcohol or drug injury to cells or inherited as a mitochondrial genetic defects. 
Table 2 Summary of genetic mitochondrial hepatopathies

\begin{tabular}{lll}
\hline Disease & Causes & Effect \\
\hline $\begin{array}{ll}\text { Neonatal Liver Failure } \\
\text { Navajo Neurohepatopathy }\end{array}$ & Defective mitochondrial gene expression in cell nucleus & Respiratory chain defects \\
$\begin{array}{l}\text { Mitochondrial DNA } \\
\text { depletion syndrome }\end{array}$ & Decreased copy number of mitochondrial DNA & Cholestasis; cirrhosis; liver failure \\
$\begin{array}{l}\text { Alpers-Huttenlocher } \\
\text { Syndrome }\end{array}$ & Defective expression of mitochondrial genes & Myopathy; hepatomegaly; progressive liver failure \\
Villous Atrophy Syndrome & Deletion in mitochondrial DNA & Hepatomegaly; jaundice; progressive coagulopathy; \\
Pearson's Syndrome & Mitochondrial DNA depletion and rearrangement & Intra-cellular respiratory dysfunction \\
\end{tabular}

Genetic mitochondrial disorders of liver: The clinically mitochondrial hepatopathies are heterogeneous and can cause acute or chronic liver failure, lactic acidosis, and cholestasis. Following are a few examples of primary mitochondrial hepatopathies caused due to mitochondrial functional defects:

1. Neonatal Liver Failure: Occurrence of acute liver failure in few weeks to months old infants due to respiratory chain defects. ${ }^{70}$ This is caused due to defective mitochondrial genes expressed in nuclear DNA. Disease is rapidly progressive and causes death within months of manifestation.

2. Navajo Neurohepatopathy: Rearrangement in mitochondrial DNA resulting in respiratory dysfunction ${ }^{71}$ causes Navajo Neurohepatopathy. Clinical symptoms include cholestasis, cirrhosis, or liver failure that occur in infancy or childhood.

3. Mitochondrial DNA depletion syndrome: This diseases is caused by decreased copy number of mitochondrial DNA and hence insufficient expression of mitochondrial genes from mitochondrial DNA. ${ }^{72}$ Besides myopathy, hepatomegaly and progressive liver failure causes death in early age.

4. Alpers-Huttenlocher Syndrome (Delayed-Onset Liver Disease): Defective expression of mitochondrial genes in cell nucleus causes this diseases. It is characterized by hepatomegaly, jaundice, and progressive coagulopathy and hypoglycemia. ${ }^{73}$. Studies show that onset of symptoms occurs between 2 months to 8 years of life.

5. Villous Atrophy Syndrome: This syndrome is caused due to deletion in mitochondrial DNA resulting in intra-cellular respiratory dysfunction. ${ }^{74}$ Symptoms include severe anorexia, vomiting, chronic diarrhea, and villus atrophy in the first year of life. Hepatic involvement is characterized by mild elevation of aminotransferases, hepatomegaly, and steatosis.

6. Pearson's Syndrome: Failure of multi organ system (hematopoietic system, exocrine pancreas, liver, and kidneys) occurs due to mitochondrial DNA depletion and rearrangement defects. Liver failure and death before the age of 4 years has been reported. ${ }^{75}$

7. Various vitamins, cofactors, respiratory substrates, or antioxidant compounds are being used to by-pass injury to the respiratory function of mitochondria in cells. ${ }^{76,77}$ Such clinical management is largely supportive and has very limited success. ${ }^{78}$ Involvement of other organ systems limits advantages of liver surgery and transplantation. ${ }^{79,80}$ Despite the extensive exploration of mitochondrial hepatopathies in recent years, much remains to be well-educated about these disorders.

Acquired mitochondrial disorders of liver: Besides mitochondrial hepatopathies caused by genetic variations, mitochondrial injuries in liver cells can also be acquired by exposure to alcohol and drugs which can cause serious damage to cells. ${ }^{69}$ Hepatocytes are more susceptible to adverse effects of these drugs since detoxification of most drugs takes place in liver. Although hepatotoxicity may be caused through a wide range of sources ${ }^{81}$ mitochondrial dysfunction is one major mechanism of injury. ${ }^{82}$ Deleterious effects of drugs on hepatic mitochondria can affect mitochondrial DNA or disturb mitochondrial normal respiratory functions. This poses major concern for pharmaceutical companies since it causes failure of a drug or withdrawal of an already marketed medicine. ${ }^{68}$ By obstructing mitochondrial respiratory function, injuring mitochondrial membrane, causing leak of pro-apoptotic proteins into cytoplasm or triggering mutations in mitochondrial DNA, drugs can cause necrosis or apoptosis of hepatocytes, leading to cytolytic hepatitis, which can further cause liver cirrhosis or failure. ${ }^{82}$ Depending on susceptibility, diverse hepatic or general symptoms, such as micro vesicular steatosis, lesions, hypoglycemia hyperlactataemia, lactic acidosis, myopathy, rhabdomyolysis, pancreatitis, peripheral neuropathy or lipoatrophy may occur. ${ }^{83,84}$ Severe liver injury instigated by mitochondrial dysfunction in clinical trials can be avoided by careful evaluation of newly developed drugs during the preclinical safety studies.

Following are examples of a few mechanism through which drugs (or their metabolites) can induce mitochondrial dysfunction:

1. Induction of damage to outer membrane of mitochondria and release of pro-apoptotic proteins: Drugs that cause damage to outer membrane of mitochondria trigger the release of pro-apoptotic proteins into cytoplasm, such as cytochrome c. Release of pro-apoptotic proteins initiates apoptosis cascades resulting in cell death. Extensive hepatic cell death may result into liver failure. For example, acetaminophen, valproic acid, salicylic acid and others can cause fulminant hepatic failure. ${ }^{85,86}$ The molecular mechanisms of induction of membrane damage by drugs are still poorly understood. Pro-apoptotic proteins from mitochondria can also be translocated indirectly from inter-membrane space to cytoplasm. ${ }^{87}$

2. Respiration enzyme impairment: Some drugs behave as mitochondrial poisons by directly impairing one or more mitochondrial enzymes required for respiration process to produce ATP ${ }^{88}$ Higher intra-mitochondrial accrual of drugs like amiodarone, perhexiline or tamoxifen causes progressive 
reduction in mitochondrial respiration. $^{88,89}$ This leads to decreased ATP production in cells.

3. Other mechanisms: Several drugs can impair other normal functions of mitochondria, such as damage to fatty acid oxidation..$^{90}$ Others can cause more than one kind of impairment to mitochondria in cells. ${ }^{82}$

Mitochondrial DNA mutation or copy number depletion: Several nucleoside based anti-viral drugs have been found to either inhibit mitochondrial DNA replication directly by inhibiting mitochondrial DNA polymerase, such as fialuridine (FIAU) ${ }^{91}$ or by incorporation and termination of growing chain of mitochondrial DNA during replication, such as stavudine (d4T), zidovudine (AZT) or didanosine (DDI) ${ }^{92,93}$ Some other toxins can manipulate replication causing point mutations or deletions/rearrangements in mitochondrial DNA.

Several environmental factors may enhance the risk of druginduced mitochondrial dysfunction. ${ }^{60,94}$ Evidence shows that alcohol abuse can induce underlying mitochondrial dysfunctions, which may render the liver more susceptible to drug-induced mitochondrial injuries. ${ }^{94}$ Some microsomal enzyme inducers or viral infections may also predispose cells to drug-induced mitochondrial damages in the liver. ${ }^{95,96}$ Release of viral proteins, cytokines and interferon can disturb intracellular homeostasis that causes additive effects to druginduced mitochondrial function impairment. ${ }^{84}$

Unlike most other differentiated cell types, hepatocytes are capable of dividing in response to liver injury. Mitochondria in hepatocytes proliferate in response to cell division and energy needs. However, if mitochondria are considerably impaired, mitochondrial division gets compromised, and cell death and potentially liver failure ensues. Aside from liver transplantation, currently there is no means available to repair mitochondrial damage, or replace dysfunctional mitochondria. Development of a method to deliver healthy and functional mitochondria to hepatocytes that can replace the dysfunctional mitochondria could help treat several mitochondrial hepatopathies caused due to direct or drug/alcohol induced damage to mitochondria.

\section{Diagnosis and treatment of mitochondrial dysfunctional disorders}

Complications in the diagnosis of mitochondrial DNA disorders arise because of lack of clear correlation between mitochondrial DNA genotype and disorder phenotype. Clinically, family history and histological variations have been found valuable to characterize mitochondrial disorders, but these are not always accurate. Molecular testing supported by clinical, histochemical, and biochemical testing has helped in development of improved rational diagnostic algorithms. ${ }^{97}$

Following are a few key histochemical and molecular approaches currently under investigations for diagnosis of mitochondrial disorders:

Histochemical and biochemical analysis: Specific histological and histochemical alterations in affected tissue of some patients has allowed to determine some respiratory complex dysfunctions in mitochondria. Accumulation of defective mitochondria with cytochrome $\mathrm{c}$ oxidase abnormalities causes appearance of raggedfibers like structures in affected tissues. Cytochrome c oxidase is an essential component of respiratory complex on inner membrane of mitochondria that takes part in ATP synthesis. ${ }^{98}$ Any abnormalities in cytochrome c oxidase cause severe metabolic dysfunctions. Raggedfibers like structures in tissues appear when mutations in mitochondrial DNA result into expression of defective subunits of cytochrome c oxidase. However tissue biopsies from patients with the MELAS phenotype or other mitochondrial DNA mutations have shown no distinct fiber like structures which limits the use of such histochemical testing. Biochemical evaluation of enzymes involved in respiratory function of mitochondria in affected tissue is also used to diagnose mitochondrial disorders in clinical labs. ${ }^{99}$ Assessment of oxygen consumption and ATP synthesis by intact organelles from affected tissue is another approach to evaluate mitochondrial function. ${ }^{100}$ The identification of specific mitochondrial enzyme abnormalities further helps to identify the particular molecular defects.

Molecular genetic analysis: Point mutations, deletions and rearrangements in mitochondrial DNA can be assayed by several genomic techniques such as Southern blotting, ${ }^{101,102}$ PCR or sequencing. ${ }^{103,104}$ Loss of normal mitochondrial DNA or accumulation of defective mitochondrial DNA is also being determined by real-time PCR in clinical labs. With the help of these molecular techniques, association of several known genes have now been established with myopathic or hepatocerebral disorders. Small size of mitochondrial DNA allows relatively easy screening of entire mitochondrial DNA for rare or novel mutations. Besides some older techniques like denaturing gradient gel electrophoresis ${ }^{102}$ and denaturing highperformance liquid chromatography, ${ }^{105}$ several new emerging technologies such as microarrays ${ }^{97}$ have proven very valuable. Newly identified mutations and variations in mitochondrial DNA should be carefully assessed and associated with human diseases because of the extreme heteroplasmic nature of the mitochondrial DNA. Though several guidelines to establish a novel pathogenic mitochondrial DNA variant have been proposed and applied in clinical studies, ${ }^{106}$ still more canonical criteria are required for accurate identification and establishment of new variants associated with human mitochondrial disorders.

1. Despite of increased understanding of mitochondrial genetics, pathogenesis of mitochondrial DNA variations and associated disorders, currently other than rare helpful cases of transplant or surgery no effective treatment options are available. Attempts for management of mitochondrial dysfunctional disorders has been made with vitamins, cofactors, metabolites or electron acceptors to bypass respiratory complex defects. ${ }^{78}$ More dedicated and diseases specific treatments are still under investigation. Following are a few approaches actively being pursued in this direction.

2. Exercise therapy: Aim of exercise therapy is to improve physical capability and quality of life in cases where myopathy is caused by mitochondrial DNA mutations. Although there is no noteworthy change in mitochondrial DNA mutations, evidence shows improved mitochondrial function. ${ }^{107}$ Enhanced ATP levels, delayed disease onset and increased life expectancy has been observed in mice undergoing muscle training exercises. ${ }^{108}$ Long-term training exercise studies are still under investigation and might help to evaluate the role of exercises on mitochondrial DNA mutations load. In another ongoing study, muscle regeneration is stimulated by proliferating undifferentiated myogenic cells in response to injury by resistance exercise. Once activated, undifferentiated myogenic cells would incorporate muscle cells with normal mitochondrial DNA which may help in shifting heteroplasmy 
levels significantly to recover muscular function in patients who have high levels of sporadically mutated mitochondrial DNA in mature muscle fibers. ${ }^{109}$ Although this approach has been proven advantageous in clinics to some levels, additional studies are required to define the optimal parameters for exercise regime. ${ }^{110}$

3. Gene therapy: Researchers are actively working on wide number of approaches to develop effective treatment of mitochondrial disorders. Manipulation of heteroplasmy levels in mitochondrial DNA such that balance of mitochondrial genome shifts from mutant to normal may also lead to decreased manifestation of mutations occurred in mitochondrial genome. Genetics-based strategies that would allow propagation of normal mitochondrial genome but inhibit the replication of mutated mitochondrial DNA are also under investigation. ${ }^{111}$ Interfering molecules are being identified that could differentiate between normal and mutated mitochondrial DNA. In another study, restriction endonucleases are being tested that could distinguish between mutated and normal mitochondrial DNA. Such approach is limited to the restriction sites generated because of mutations in DNA. ${ }^{112}$ Mutated mitochondrial DNA is also being selectively targeted with zinc finger DNA methylase which can bind the DNA in a sequencespecific manner and modify or cleave the mutated genome in mitochondria. ${ }^{113}$ Major obstacle in these strategies is delivery of required molecules in matrix of mitochondria across the two mitochondrial membranes. Further investigations are ongoing to establish process of delivery and the ability of such molecules to hinder mutated mitochondrial DNA replication, and their efficiency in vivo.

Similar to many of mitochondrial proteins that are expressed in nucleus and transported to mitochondria, expression and transport of mitochondrial genes in nucleus which are mutated in mitochondrial DNA and causing mitochondria functional defects may help to recover normal functions of mitochondria. ${ }^{114}$. Difficulties in mitochondrial localization of such nuclear expressions are anticipated. ${ }^{115}$ Recently a mechanism has been demonstrated by which cytosolic t-RNA could be imported in mitochondria. ${ }^{116}$ Such techniques may assist in treatment of disorders caused due to defects in genes expressing mitochondrial t-RNAs. Taken together, ongoing investigations to develop successful gene therapies for treatment of mitochondrial disorders are encouraging.

In another study to develop a potential treatment for mitochondrial disorders, reprogrammed cells derived from patients with dysfunctional mitochondria were implanted to repopulate cells with normal mitochondria in tissues. ${ }^{117}$ Induced pluripotent stem cells (iPSC) exhibiting normal mitochondrial functions were also screened from a population of iPSCs derived from affected patients. Pluripotent cells were also generated by somatic cell nuclear transfer, that is replacement of nuclear DNA of the egg with nuclear DNA of the diseased somatic cells, and there by another method to obtain potential cells containing healthy mitochondria that can be used to correct mitochondrial disorders. Mitochondrial DNA mutations transmission from mother to offspring vary considerably ${ }^{118}$ which creates challenges for providing the genetic counseling to affected families. Though prenatal genetic diagnosis may detect mitochondrial DNA mutation load, clinically major concern arises if heteroplasmy levels reflect phenotypic outcomes at other fetal tissue levels. ${ }^{119}$ Currently, mitochondrial gene replacement methods offer great potential for development of techniques to prevent human mitochondrial DNA disease transmission. Transfer of the pronuclei from a fertilized zygote of a female mouse with mitochondrial DNA disease to an enucleated zygote from a healthy female donor has produced healthy offspring successfully. ${ }^{120}$ Recently United Kingdom approved a genetherapy technique, in which the nucleus from a human female egg is transferred to a donor enucleated egg with healthy mitochondria, followed by fertilization of the donor egg. ${ }^{121,122}$

Development of study models: Since variations or other environmental factors can be selectively deleterious for mitochondria in some individuals, it may also be important to use novel study models with underlying mitochondrial and/or metabolic abnormalities. This could help in improved prediction of characteristics of injury and development of potential treatments. Pathophysiology of the mitochondrial dysfunction will have to be carefully considered in such study models. ${ }^{123,124}$

\section{Conclusion}

Several innate and acquired abnormalities may cause inadequate functions of mitochondria that may result in cell death. While variable penetrance and expression of mitochondrial mutations makes the clinical identification and significance of variations more challenging, several targeted population based studies have provided insights into systematic association of mitochondrial abnormalities to clinical syndromes. Despite significant progress in understanding and documenting the mitochondrial DNA defects causing mitochondrial dysfunctions, diagnosis of disorders have become more challenging with increased number of reported pathogenic mutations and constantly evolving guidelines of pathogenicity. Novel experimental approaches for high-throughput screening and mitochondrial DNA disease treatment under investigation are promising. Moreover, research strategies focused on elucidating the molecular mechanisms of mitochondrial dysfunction and complex relationships among mitochondrial DNA variations and associated diverse clinical phenotypes have been also useful in developing therapies for mitochondrial disorders. Though establishing the pathogenesis of mitochondrial disorders will uncover novel targets or molecular pathways that may be exploited for therapies development, meanwhile, approaches to develop generic treatment methods for mitochondrial disorders should be actively pursued. Evidences from current studies under investigation will also provide the foundation for development of targeted therapeutic approaches for mitochondrial disorders.

\section{Acknowledgements}

None.

\section{Conflict of interest}

The author declares no conflict of interest.

\section{References}

1. Hayashi J, Takemitsu M, Goto Y, et al. Human mitochondria and mitochondrial genome function as a single dynamic cellular unit. $J$ Cell Biol. 1994;125(1):43-50.

2. Liu YJ, Mason DY, Johnson GD, et al. Germinal center cells express bcl-2 protein after activation by signals which prevent their entry into apoptosis. Eur J Immunol. 1991;21(8):1905-1910.

3. Tait SW, Green DR. Mitochondria and cell signalling. J Cell Sci. 2012;125(Pt 4):807-815. 
4. Zhdanov AV, Okkelman IA, Collins FW, et al. A novel effect of DMOG on cell metabolism: direct inhibition of mitochondrial function precedes HIF target gene expression. Biochim Biophys Acta. 2015;1847(10):1254 1266.

5. Mandal S, Lindgren AG, Srivastava AS, et al. Mitochondrial function controls proliferation and early differentiation potential of embryonic stem cells. Stem Cells. 2011;29(3):486-495.

6. Lartigue L, Kushnareva Y, Seong Y, et al. Caspase-independent mitochondrial cell death results from loss of respiration, not cytotoxic protein release. Mol Biol Cell. 2009;20(23):4871-4884.

7. Karbowski M, Youle RJ. Regulating mitochondrial outer membrane proteins by ubiquitination and proteasomal degradation. Curr Opin Cell Biol. 2011;23(4):476-482.

8. Colbeau A, Nachbaur J, Vignais PM. Enzymic characterization and lipid composition of rat liver subcellular membranes. Biochim Biophys Acta. 1971;249(2):462-492.

9. Weeber EJ, Levy M, Sampson MJ, et al. The role of mitochondrial porins and the permeability transition pore in learning and synaptic plasticity. $J$ Biol Chem. 2002;277(21):18891-18897.

10. Klingenberg M. The ADP and ATP transport in mitochondria and its carrier. Biochim Biophys Acta. 2008;1778(10):1978-2021.

11. Nargang FE, Rapaport D, Ritzel RG, et al. Role of the negative charges in the cytosolic domain of TOM22 in the import of precursor proteins into mitochondria. Mol Cell Biol. 1998;18(6):3173-3181.

12. Volman H. A morphologic and morphometric study of the mitochondria in several hepatoma cell lines and in isolated hepatocytes. Virchows Arch B Cell Pathol. 1978;26(3):249-259.

13. Comte J, Maisterrena B, Gautheron DC. Lipid composition and protein profiles of outer and inner membranes from pig heart mitochondria Comparison with microsomes. Biochim Biophys Acta. 1976;419(2):271284

14. King ME, GC Godman, King DW. Respiratory enzymes and mitochondria morphology of HeLa and L cells treated with chloramphenicol and ethidium bromide. J Cell Biol. 1972;53(1):127-142.

15. Birch-Machin MA, Turnbull DM. Assaying mitochondrial respiratory complex activity in mitochondria isolated from human cells and tissues. Methods Cell Biol. 2001;65:97-117.

16. Van der Bliek AM, Shen Q, Kawajiri S. Mechanisms of mitochondrial fission and fusion. Cold Spring Harb Perspect Biol. 2013:5(6).

17. Collins TJ, Berridge MJ, Lipp P, et al. Mitochondria are morphologically and functionally heterogeneous within cells. EMBO J. 2002;21(7):16161627.

18. Westermann B. Bioenergetic role of mitochondrial fusion and fission Biochim Biophys Acta. 2012;1817(10):1833-1838.

19. Imoto $M$, Tachibana I, Urrutia R. Identification and functiona characterization of a novel human protein highly related to the yeast dynamin-like GTPase Vps1p. J Cell Sci. 1998;111(Pt 10):1341-1349.

20. Tieu Q, Nunnari J. Mdv1p is a WD repeat protein that interacts with the dynamin-related GTPase, Dnm1p, to trigger mitochondrial division. $J$ Cell Biol. 2000;151(2):353-366.

21. Labrousse AM, Zappaterra MD, Rube DA, et al. C. elegans dynaminrelated protein DRP-1 controls severing of the mitochondrial outer membrane. Mol Cell. 1999;4(5):815-826.

22. Mozdy AD, McCaffery JM, Shaw JM. Dnm1p GTPase-mediated mitochondrial fission is a multi-step process requiring the novel integral membrane component Fis1p. J Cell Biol. 2000;151(2):367-380.
23. Shin-ya Miyagishima, RI, Kyoko Toda, Haruko Kuroiwa, et al. Realtime analyses of chloroplast and mitochondrial division and differences in the behavior of their dividing rings during contraction. Planta Springer-Verlag; 1999. 207(3):343-353.

24. Shepard KA, Yaffe MP. The yeast dynamin-like protein, Mgm1p, functions on the mitochondrial outer membrane to mediate mitochondrial inheritance. J Cell Biol. 1999;144(4):711-720.

25. Alexander C, Votruba M, Pesch UE, et al. OPA1, encoding a dynaminrelated GTPase, is mutated in autosomal dominant optic atrophy linked to chromosome 3q28. Nat Genet. 2000;26(2):211-215.

26. Rapaport D, Brunner M, Neupert W, et al. Fzolp is a mitochondrial outer membrane protein essential for the biogenesis of functional mitochondria in Saccharomyces cerevisiae. J Biol Chem. 1998;273(32):20150-201555.

27. Walberg MW, Clayton DA. In vitro transcription of human mitochondria DNA. Identification of specific light strand transcripts from the displacement loop region. J Biol Chem. 1983;258(2):1268-1275.

28. Kunisada T, Yamagishi H. Sequence repetition and genomic distribution of small polydisperse circular DNA purified from HeLa cells. Gene. $1984 ; 31(1-3): 213-223$

29. Bonawitz ND, Clayton DA, Shadel GS. Initiation and beyond: multiple functions of the human mitochondrial transcription machinery. Mol Cell. 2006;24(6):813-825.

30. Alberts B JA, Lewis J, Martin Raff, et al. Molecular Biology of the Cell The Mitochondrion. 4th ed. USA: Garland Science; 2002.

31. Tessman I, Kennedy MA. DNA polymerase II of Escherichia coli in the bypass of abasic sites in vivo. Genetics. 1994;136(2):439-448.

32. Clayton DA, Doda JN, Friedberg EC. Absence of a pyrimidine dimer repair mechanism for mitochondrial DNA in mouse and human cells. Basic Life Sci. 1975;5B:589-591.

33. Longley MJ, Nguyen D, Kunkel TA, et al. The fidelity of human DNA polymerase gamma with and without exonucleolytic proofreading and the p55 accessory subunit. J Biol Chem. 2001;276(42):38555-38562.

34. Thorslund T, Sunesen M, Bohr VA, et al. Repair of 8-oxoG is slower in endogenous nuclear genes than in mitochondrial DNA and is without strand bias. DNA Repair (Amst). 2002;1(4):261-273.

35. He Y, Wu J, Dressman DC, et al. Heteroplasmic mitochondrial DNA mutations in normal and tumour cells. Nature. 2010;464(7288):610-614.

36. Limongelli A, Schaefer J, Jackson S, et al. Variable penetrance of a familial progressive necrotising encephalopathy due to a novel tRNA(Ile) homoplasmic mutation in the mitochondrial genome. $J \mathrm{Med}$ Genet. 2004;41(5):342-349.

37. Nakada K, Inoue K, Ono T, et al. Inter-mitochondrial complementation: Mitochondria-specific system preventing mice from expression of disease phenotypes by mutant mtDNA. Nat Med. 2001;7(8):934-940.

38. Ivanov PL, Wadhams MJ, Roby RK, et al. Mitochondrial DNA sequence heteroplasmy in the Grand Duke of Russia Georgij Romanoy establishes the authenticity of the remains of Tsar Nicholas II. Nat Genet. 1996;12(4):417-420.

39. Kocher TD, Thomas WK, Meyer A, et al. Dynamics of mitochondrial DNA evolution in animals: amplification and sequencing with conserved primers. Proc Natl Acad Sci U S A. 1989;86(16):6196-6200.

40. Hall PA, Coates PJ, Ansari B, et al. Regulation of cell number in the mammalian gastrointestinal tract: the importance of apoptosis. J Cell Sci. 1994;107(Pt 12):3569-3577.

41. Lawson JA, Fisher MA, Simmons CA, et al. Inhibition of Fas receptor (CD95)-induced hepatic caspase activation and apoptosis by acetaminophen in mice. Toxicol Appl Pharmacol. 1999;156(3):179-186. 
42. Liu X, Kim CN, Yang J, et al. Induction of apoptotic program in cell-free extracts: requirement for dATP and cytochrome c. Cell. 1996;86(1):147157

43. Hao Z, Duncan GS, Chang CC, et al. Specific ablation of the apoptotic functions of cytochrome $\mathrm{C}$ reveals a differential requirement for cytochrome C and Apaf-1 in apoptosis. Cell. 2005;121(4):579-591.

44. Aouacheria A, Cibiel A, Guillemin Y, et al. Modulating mitochondriamediated apoptotic cell death through targeting of Bcl-2 family proteins. Recent Pat DNA Gene Seq. 2007;1(1):43-61.

45. Boland ML, Chourasia AH, Macleod KF. Mitochondrial dysfunction in cancer. Front Oncol. 2013;3:292.

46. Lee WS, Sokol RJ. Liver disease in mitochondrial disorders. Semin Liver Dis. 2007;27(3):259-273.

47. Meyers DE, Basha HI, Koenig MK. Mitochondrial cardiomyopathy: pathophysiology, diagnosis, and management. Tex Heart Inst J. 2013;40(4):385-394.

48. Haas RH. Autism and mitochondrial disease. Dev Disabil Res Rev. 2010;16(2):144-153.

49. Moraes CT, DiMauro S, Zeviani M, et al. Mitochondrial DNA deletions in progressive external ophthalmoplegia and Kearns-Sayre syndrome. $N$ Engl J Med. 1989;320(20):1293-1299.

50. Treem WR, Sokol RJ. Disorders of the mitochondria. Semin Liver Dis. 1998;18(3):237-253.

51. Weber K, Wilson JN, Taylor L, et al. A new mtDNA mutation showing accumulation with time and restriction to skeletal muscle. Am J Hum Genet. 1997;60(2):373-380.

52. Sacconi S, Salviati L, Nishigaki Y, et al. A functionally dominant mitochondrial DNA mutation. Hum Mol Genet. 2008;17(12):1814-1820.

53. Cortopassi GA, Shibata D, Soong NW, et al. A pattern of accumulation of a somatic deletion of mitochondrial DNA in aging human tissues. Proc Natl Acad Sci U S A. 1992;89(16):7370-7374.

54. Bender A, Krishnan KJ, Morris CM, et al. High levels of mitochondrial DNA deletions in substantia nigra neurons in aging and Parkinson disease. Nat Genet. 2006;38(5):515-517.

55. Rahman S, Poulton J. Diagnosis of mitochondrial DNA depletion syndromes. Arch Dis Child. 2009;94(1):3-5.

56. Gorman GS, Schaefer AM, Ng Y, et al. Prevalence of nuclear and mitochondrial DNA mutations related to adult mitochondrial disease. Ann Neurol. 2015;77(5):753-759.

57. Schneider S, Excoffier L. Estimation of past demographic parameters from the distribution of pairwise differences when the mutation rates vary among sites: application to human mitochondrial DNA. Genetics. 1999;152(3):1079-1089

58. Reeve AK, Krishnan KJ, Turnbull D. Mitochondrial DNA mutations in disease, aging, and neurodegeneration. Ann N Y Acad Sci. 2008;1147:2129.

59. Chiu AY, Zhai P, Dal Canto MC, et al. Age-dependent penetrance of disease in a transgenic mouse model of familial amyotrophic lateral sclerosis. Mol Cell Neurosci. 1995;6(4):349-362.

60. McCormack AL, Thiruchelvam M, Manning-Bog AB, et al. Environmental risk factors and Parkinson's disease:selective degeneration of nigral dopaminergic neurons caused by the herbicide paraquat. Neurobiol Dis. 2002;10(2):119-127.

61. Chol M, Lebon S, Bénit P, et al. The mitochondrial DNA G13513A MELAS mutation in the NADH dehydrogenase 5 gene is a frequent cause of Leigh-like syndrome with isolated complex I deficiency. $J$ Med Genet. 2003;40(3):188-191.
62. Goto Y, Nonaka I, Horai S. A mutation in the tRNA(Leu)(UUR) gene associated with the MELAS subgroup of mitochondrial encephalomyopathies. Nature. 1990;348(6302):651-653.

63. Macmillan $\mathrm{C}$, Kirkham $\mathrm{T}, \mathrm{Fu} \mathrm{K}$, et al. Pedigree analysis of French Canadian families with T14484C Leber's hereditary optic neuropathy. Neurology. 1998;50(2):417-422.

64. Man PY, Griffiths PG, Brown DT, et al. The epidemiology of Leber hereditary optic neuropathy in the North East of England. Am J Hum Genet. 2003;72(2):333-339.

65. Shoffner JM, Lott MT, Lezza AM, et al. Myoclonic epilepsy and raggedred fiber disease (MERRF) is associated with a mitochondrial DNA tRNA(Lys) mutation. Cell. 1990;61(6):931-937.

66. Taylor RW, Schaefer AM, Barron MJ, et al. The diagnosis of mitochondrial muscle disease. Neuromuscul Disord. 2004;14(4):237-245.

67. Degli Esposti D, Hamelin J, Bosselut N, et al. Mitochondrial roles and cytoprotection in chronic liver injury. Biochem Res Int. 2012;2012:387626.

68. Labbe G, Pessayre D, Fromenty B. Drug-induced liver injury through mitochondrial dysfunction: mechanisms and detection during preclinical safety studies. Fundam Clin Pharmacol. 2008;22(4):335-353.

69. Chariot P, Drogou I, de Lacroix-Szmania I, et al. Zidovudine-induced mitochondrial disorder with massive liver steatosis, myopathy, lactic acidosis, and mitochondrial DNA depletion. J Hepatol. 1999;30(1):156160 .

70. Cormier-Daire V, Chretien D, Rustin P, et al. Neonatal and delayedonset liver involvement in disorders of oxidative phosphorylation. $J$ Pediatr. 1997;130(5):817-822.

71. Holve S, Hu D, Shub M, et al. Liver disease in Navajo neuropathy. $J$ Pediatr. 1999;135(4):482-493.

72. Moraes CT, Shanske S, Tritschler HJ, et al. mtDNA depletion with variable tissue expression: a novel genetic abnormality in mitochondrial diseases. Am J Hum Genet. 1991;48(3):492-501.

73. Narkewicz MR, Sokol RJ, Beckwith B, et al. Liver involvement in Alpers disease. J Pediatr. 1991;119(2):260-267.

74. Cormier-Daire V, Bonnefont JP, Rustin P, et al. Mitochondrial DNA rearrangements with onset as chronic diarrhea with villous atrophy. $J$ Pediatr. 1994;124(1):63-70.

75. Morikawa Y, Matsuura N, Kakudo K, et al. Pearson's marrow/pancreas syndrome: a histological and genetic study. Virchows Arch A Pathol Anat Histopathol. 1993;423(3):227-231.

76. Mahoney DJ, Parise G, Tarnopolsky MA. Nutritional and exercise-based therapies in the treatment of mitochondrial disease. Curr Opin Clin Nutr Metab Care. 2002;5(6):619-629.

77. Eleff S, Kennaway NG, Buist NR, et al. 31P NMR study of improvement in oxidative phosphorylation by vitamins $\mathrm{K} 3$ and $\mathrm{C}$ in a patient with a defect in electron transport at complex III in skeletal muscle. Proc Natl Acad Sci U S A. 1984;81(11):3529-3533.

78. Chen RS, Huang CC, Chu NS. Coenzyme Q10 treatment in mitochondrial encephalomyopathies. Short-term double-blind, crossover study. Eur Neurol. 1997;37(4):212-218.

79. Dubern B, Broue P, Dubuisson C, et al. Orthotopic liver transplantation for mitochondrial respiratory chain disorders: a study of 5 children. Transplantation. 2001;71(5):633-637.

80. Thomson M, McKiernan P, Buckels J, et al. Generalised mitochondrial cytopathy is an absolute contraindication to orthotopic liver transplant in childhood. J Pediatr Gastroenterol Nutr. 1998;26(4):478-481. 
81. Huang YS, Chern HD, Su WJ, et al. Cytochrome P450 2E1 genotype and the susceptibility to antituberculosis drug-induced hepatitis. Hepatology. 2003;37(4):924-930.

82. Fromenty B, Pessayre D. Inhibition of mitochondrial beta-oxidation as a mechanism of hepatotoxicity. Pharmacol Ther. 1995;67(1):101-154.

83. Note R, Maisonneuve C, Lettéron P, et al. Mitochondrial and metabolic effects of nucleoside reverse transcriptase inhibitors (NRTIs) in mice receiving one of five single- and three dual-NRTI treatments. Antimicrob Agents Chemother. 2003;47(11):3384-3392.

84. Gougeon ML, Pénicaud L, Fromenty B, et al. Adipocytes targets and actors in the pathogenesis of HIV-associated lipodystrophy and metabolic alterations. Antivir Ther. 2004;9(2):161-177.

85. Masubuchi Y, Kano S, Horie T. Mitochondrial permeability transition as a potential determinant of hepatotoxicity of antidiabetic thiazolidinediones Toxicology. 2006;222(3):233-239.

86. Trost LC, Lemasters. The mitochondrial permeability transition: a new pathophysiological mechanism for Reye's syndrome and toxic liver injury. J Pharmacol Exp Ther. 1996;278(3):1000-1005.

87. Bae MA, Song BJ. Critical role of c-Jun N-terminal protein kinase activation in troglitazone-induced apoptosis of human HepG2 hepatoma cells. Mol Pharmacol. 2003;63(2):401-408.

88. Berson A, De Beco V, Lettéron P, et al. Steatohepatitis-inducing drugs cause mitochondrial dysfunction and lipid peroxidation in rat hepatocytes. Gastroenterology. 1998;114(4):764-774.

89. Larosche I, Lettéron P, Fromenty B, et al. Tamoxifen inhibits topoisomerases, depletes mitochondrial DNA, and triggers steatosis in mouse liver. J Pharmacol Exp Ther. 2007;321(2):526-535.

90. Deschamps D, Fisch C, Fromenty B, et al. Inhibition by salicylic acid of the activation and thus oxidation of long chain fatty acids. Possible role in the development of Reye's syndrome. $J$ Pharmacol Exp Ther. 1991;259(2):894-904.

91. Lewis W, Levine ES, Griniuviene B, et al. Fialuridine and its metabolites inhibit DNA polymerase gamma at sites of multiple adjacent analog incorporation, decrease mtDNA abundance, and cause mitochondrial structural defects in cultured hepatoblasts. Proc Natl Acad Sci U S A. 1996;93(8):3592-3597.

92. Benbrik E, Chariot P, Bonavaud S, et al. Cellular and mitochondrial toxicity of zidovudine (AZT), didanosine (ddI) and zalcitabine (ddC) on cultured human muscle cells. J Neurol Sci. 1997;149(1):19-25.

93. Maisonneuve C, Igoudjil A, Begriche K, et al. Effects of zidovudine, stavudine and beta-aminoisobutyric acid on lipid homeostasis in mice: possible role in human fat wasting. Antivir Ther. 2004;9(5):801-810.

94. Fromenty B, Grimbert S, Mansouri A, et al. Hepatic mitochondrial DNA deletion in alcoholics:association with microvesicular steatosis Gastroenterology. 1995;108(1):193-200.

95. Levy M. Role of viral infections in the induction of adverse drug reactions. Drug Saf. 1997;16(1):1-8.

96. Prandota J. Important role of prodromal viral infections responsible for inhibition of xenobiotic metabolizing enzymes in the pathomechanism of idiopathic Reye's syndrome, Stevens-Johnson syndrome, autoimmune hepatitis, and hepatotoxicity of the therapeutic doses of acetaminophen used in genetically predisposed persons. Am J Ther. 2002;9(2):149-156

97. Zhou S, Kassauei K, Cutler DJ, et al. An oligonucleotide microarray for high-throughput sequencing of the mitochondrial genome. J Mol Diagn. 2006;8(4):476-482.

98. Szuhai K, Ouweland J, Dirks R, et al. Simultaneous A8344G heteroplasmy and mitochondrial DNA copy number quantification in myoclonus epilepsy and ragged-red fibers (MERRF) syndrome by a multiplex molecular beacon based real-time fluorescence PCR. Nucleic Acids Res. 2001;29(3):E13.
99. Kirby DM, Thorburn DR, Turnbull DM, et al. Biochemical assays of respiratory chain complex activity. Methods Cell Biol. 2007;80:93-119.

100. Janssen AJ, Schuelke M, Smeitink JA, et al. Muscle $3243 \mathrm{~A} \rightarrow \mathrm{G}$ mutation load and capacity of the mitochondrial energy-generating system. Ann Neurol. 2008;63(4):473-481.

101. Holt IJ, Harding AE, Morgan-Hughes JA. Deletions of muscle mitochondrial DNA. Nature. 1988;331(6158):717-719.

102. Fischer SG, Lerman LS. Length-independent separation of DNA restriction fragments in two-dimensional gel electrophoresis. Cell. 1979;16(1):191-200

103. Holt IJ, Harding AE, Morgan-Hughes JA. Deletions of muscle mitochondrial DNA in mitochondrial myopathies: sequence analysis and possible mechanisms. Nucleic Acids Res. 1989;17(12):4465-4469.

104. Taylor RW, Taylor GA, Durham SE, et al. The determination of complete human mitochondrial DNA sequences in single cells: implications for the study of somatic mitochondrial DNA point mutations. Nucleic Acids Res. 2001;29(15):E74.

105. Van Den Bosch BJ, de Coo RF, Scholte HR, et al. Mutation analysis of the entire mitochondrial genome using denaturing high performance liquid chromatography. Nucleic Acids Res. 2000;28(20):E89.

106. McFarland R, Elson JL, Taylor RW, et al. Assigning pathogenicity to mitochondrial tRNA mutations: when "definitely maybe" is not good enough. Trends Genet. 2004;20(12):591-596.

107. Taivassalo T, Gardner JL, Taylor RW, et al. Endurance training and detraining in mitochondrial myopathies due to single large-scale mtDNA deletions. Brain. 2006;129(Pt 12):3391-3401.

108. Wenz T, Diaz F, Hernandez D, et al. Endurance exercise is protective for mice with mitochondrial myopathy. J Appl Physiol (1985). 2009;106(5):1712-1719.

109. Murphy JL, Blakely EL, Schaefer AM, et al. Resistance training in patients with single, large-scale deletions of mitochondrial DNA. Brain. 2008;131(Pt 11):2832-2840.

110. Taivassalo T, Fu K, Johns T, et al. Gene shifting: a novel therapy for mitochondrial myopathy. Hum Mol Genet. 1999;8(6):1047-1052.

111. Taylor RW, Chinnery PF, Turnbull DM, et al. Selective inhibition of mutant human mitochondrial DNA replication in vitro by peptide nucleic acids. Nat Genet. 1997;15(2):212-215.

112. Bacman SR, Chinnery PF, Turnbull DM, et al. Modulating mtDNA heteroplasmy by mitochondria-targeted restriction endonucleases in a 'differential multiple cleavage-site' model. Gene Ther. 2007;14(18):1309-1318.

113. Minczuk M, Papworth MA, Kolasinska P, et al. Sequence-specific modification of mitochondrial DNA using a chimeric zinc finger methylase. Proc Natl Acad Sci U S A. 2006;103(52):19689-19694.

114. Manfredi G, Fu J, Ojaimi J, et al. Rescue of a deficiency in ATP synthesis by transfer of MTATP6, a mitochondrial DNA-encoded gene, to the nucleus. Nat Genet. 2002;30(4):394-349.

115. Bonnet C, Kaltimbacher V, Ellouze S, et al. Allotopic mRNA localization to the mitochondrial surface rescues respiratory chain defects in fibroblasts harboring mitochondrial DNA mutations affecting complex I or v subunits. Rejuvenation Res. 2007;10(2):127-144.

116. Rubio MA, Rinehart JJ, Krett B, et al. Mammalian mitochondria have the innate ability to import tRNAs by a mechanism distinct from protein import. Proc Natl Acad Sci U S A. 2008;105(27):9186-9191.

117. Ma H, Folmes CD, Wu J, et al. Metabolic rescue in pluripotent cells from patients with mtDNA disease. Nature. 2015;524(7564):234-238.

118. Brown DT, Samuels DC, Michael EM, et al. Random genetic drift determines the level of mutant mtDNA in human primary oocytes. $\mathrm{Am} \mathrm{J}$ Hum Genet. 2001;68(2):533-536. 
119. White SL, Shanske S, Biros I, et al. Two cases of prenatal analysis for the pathogenic $\mathrm{T}$ to $\mathrm{G}$ substitution at nucleotide 8993 in mitochondrial DNA. Prenat Diagn. 1999;19(12):1165-1168.

120. Sato A, Kono T, Nakada K, et al. Gene therapy for progeny of mito-mice carrying pathogenic mtDNA by nuclear transplantation. Proc Natl Acad Sci U S A. 2005;102(46):16765-16770.

121. Craven L, Tuppen HA, Greggains GD, et al. Pronuclear transfer in human embryos to prevent transmission of mitochondrial DNA disease. Nature. 2010;465(7294):82-85.
122. Callaway E. World hails embryo vote. Nature. 2015;518(7538):145-146.

123. Greengard O, Federman M, Knox WE. Cytomorphometry of developing rat liver and its application to enzymic differentiation. J Cell Biol. 1972;52(2):261-272

124. Ruepp SU, Tonge RP, Shaw J, et al. Genomics and proteomics analysis of acetaminophen toxicity in mouse liver. Toxicol Sci. 2002;65(1):135-150. 